\title{
Oral Human Papillomavirus Infection in Children during the First 6 Years of Life, Finland
}

Stina Syrjänen, Marjut Rintala, Marja Sarkola, Jaana Willberg, Jaana Rautava, Hanna Koskimaa, Anna Paaso, Kari Syrjänen, Seija Grénman, ${ }^{1}$ Karolina Louvanto ${ }^{1}$

Human papillomavirus (HPV) infections are found in children, but transmission modes and outcomes are incompletely understood. We evaluated oral samples from 331 children in Finland who participated in the Finnish Family HPV Study from birth during 9 follow-up visits (mean time 51.9 months). We tested samples for 24 HPV genotypes. Oral HPV prevalence for children varied from $8.7 \%$ (at a 36 -month visit) to $22.8 \%$ (at birth), and $18 \mathrm{HPV}$ genotypes were identified. HPV16 was the most prevalent type to persist, followed by HPV18, HPV33, and HPV6. Persistent, oral, high-risk HPV infection for children was associated with oral HPV carriage of the mother at birth and seroconversion of the mother to high-risk HPV during follow-up (odds ratio 1.60-1.92, 95\% Cl 1.02-2.74). Children acquire their first oral HPV infection at an early age. The HPV status of the mother has a major impact on the outcome of oral HPV persistence for her offspring.

$\mathrm{C}$ utaneous warts are common in children and are acquired mostly through horizontal transmission, but also through vertical transmission; lesions can persist asymptomatically for years (1). Unlike human papillomavirus (HPV) infections of the skin, mucosal HPV infections have mostly been regarded as sexually transmitted diseases. However, certain mucosal HPVs ( $\alpha$-HPVs) have also been found in virgins, infants, and children in oral and genital mucosa, implicating a nonsexual mode of transmission (2-6). From the clinical point of view, virus clades 7, 9, and 10, which include high-risk HPV genotypes, are the major subgroups of a-HPVs. These high-risk HPVs are known to be involved in development of anogenital and head and neck cancers, and estimated to be causally associated with $\approx 4.5 \%$ of all human cancers (7).

Author affiliations: University of Turku, Turku, Finland (S. Syrjänen, M. Rintala, J. Willberg, J. Rautava, H. Koskimaa, A. Paaso,

S. Grenman, K. Louvanto); Central Hospital of Lahti, Lahti, Finland (M. Sarkola); Biohit Oyj, Helsinki, Finland (K. Syrjänen)

DOI: https://doi.org/10.3201/eid2703.202721
Nonsexual HPV transmission modes includes vertical or horizontal transmission and autoinoculation (i.e., multisite HPV infections, which can spread from 1 site to another within an individual). Vertical transmission can be further categorized as periconceptual (time around fertilization), prenatal (during pregnancy), and perinatal (during birth or immediately thereafter) $(3,8)$. Perinatal transmission has been regarded as the most likely explanation for HPV detection in newborns. Several studies have shown that children born to HPV-positive mothers have a higher risk of becoming HPV positive (9-14). Meta-analysis of 3,128 mother-child pairs showed that children born to HPV-positive mothers were $33 \%$ more likely to be HPV positive than children born to HPV-negative mothers (6). This risk was even higher $(45 \%)$ when only high-risk HPV infections were considered (6).

Periconceptual transmission could theoretically occur through infected oocytes or spermatozoa. HPV DNA has been detected in semen, sperm, seminal plasma, spermatozoa, and vas deferens (15). Studies have shown that the placenta is not a sterile microenvironment; instead, it has been shown to harbor both viruses and bacteria, which can further influence the maternal part of periconceptual transmission (16). HPV has been found in the placenta and shown to replicate in trophoblasts, which could feasibly explain prenatal transmission (2). A recent systematic review on intrauterine HPV transmission showed that the pooled percentage of antenatal vertical HPV transmission was 4.9\% $(95 \%$ CI $1.65 \%-9.85 \%)$, and the mode of delivery had no effect on this transmission (17).

Elucidation of the early HPV infections is needed to generate a comprehensive overview on the natural history of HPV infections. The main aims of this study were to characterize oral HPV prevalence and genotype variation in children in Finland and determine infection outcomes during the first 6 years of life.

${ }^{1}$ These senior authors contributed equally to this article. 


\section{Material and Methods}

\section{Participants}

The Finnish Family HPV Study is a prospective cohort study conducted at the University of Turku and Turku University Hospital, Turku, Finland, since 1998. Members of 329 families were enrolled (329 mothers, 131 fathers, and 331 newborns) as described $(13,14,18)$. Women were enrolled at a minimum of 36 weeks of their index pregnancy and subsequently followed up for 6 years. HPV status of mothers was not available before enrollment. All parents provided written, informed consent at the first visit for the study. The Research Ethics Committee of Turku University Hospital approved the study protocol and its amendments (\#2/1998 and \#2/2006).

We collected demographic data from parents by using structured questionnaires at baseline and at 3 -year and 6-year visits. General health of children was recorded at the 36-month visit (Appendix Table 1, https://wwwnc.cdc.gov/EID/article/27/3/202721-App1.pdf), and examination of oral mucosa was performed at the 6-year follow-up visit.

\section{Samples and HPV Genotyping}

Oral scrapings for HPV testing were obtained at birth; at day 3 before leaving the hospital; and at 1-, 2-, 6-, 12-, 24-, and 36-month and 6-year follow-up visits. Oral scrapings were obtained by using a brush (Cytobrush; MedScan Medical AB, https://www.diapath. com) and covering the entire oral mucosa as described (13). HPV DNA was extracted from oral scrapings by using the high salt method, as described (13). For HPV testing, we used nested PCR (MY09/MY11 external primers and GP05+/bioGP06+ internal primers) because the viral load/cell and the number of infected cells among uninfected cells was expected to be low in oral samples.

After nested PCR, HPV genotyping was performed by using the Multimetrix Kit (Progen Biotechnik GmbH, https://www.progen.com), which detected 24 low-risk, putative high-risk, and highrisk HPV genotypes as follows: 6 low-risk genotypes (HPV6, 11, 42, 43, 44, and 70); 3 putative high-risk genotypes (HPV26, 53 and 66); and 15 high-risk genotypes (HPV16, 18, 31, 33, 35, 39, 45, 51, 52, 56, 58, 59, 68, 73, and 82 (19). Blood samples from the mother and father were taken at baseline and at 12,24, and 36 months of the follow-up and stored as described (20). Antibodies to the major capsid protein L1 of HPV6, $11,16,18$, and 45 were analyzed by using multiplex HPV serologic analysis based on glutathione S-transferase fusion protein capture on fluorescent beads, as described (21). Serum samples were scored as positive when antigen-specific medium fluorescence intensity values exceeded the cutoff level of 200 for L1 antigen of individual HPV types.

\section{Statistical Analysis}

Times in months to incident oral HPV infections were calculated from the baseline visit to the first incident event. Genotype-specific HPV persistence was recorded whenever $\geq 2$ consecutive follow-up samples were positive for the same individual HPV genotype as a single infection or as part of a multiple-type infection. Clearance was defined as an event at any follow-up visit for which a previously HPV-positive test result turned out to be negative and remained HPV negative to the end of the follow-up. Times in months to the first clearance event were calculated as the time of the first visit by an HPV-positive patient to the first clearance event.

Predictors of incident HPV infection and genotype-specific HPV clearance or persistence were analyzed by using the most prevalent high-risk HPV types (species a7: HPV18, 39, 45, 59, 68, and 70; species a9: HPV16, 31 ,33, 35, 52, and 58). To model incident infections and genotype-specific HPV clearance, Poisson regression analysis was used. For persistence, a generalized estimating equation (GEE) modeling was used. In the univariate GEE model, all covariates recorded at baseline and previously implicated as potential risk factors for HPV infections were tested $(13,14)$. The following risk factors were analyzed for the both parents: age; age at time of first sexual encounter; number of lifetime sexual partners; smoking; use of alcohol; history of skin warts, oral/genital warts, and papillomas; history of sexually transmitted infections; drug consumption; oral and genital HPV DNA status; and HPV serologic results at baseline before the birth of the index child. For the mother, the risk factors were a Pap test at baseline, delivery mode, rupture of membrane, and breast-feeding. In the multivariate GEE model, only variables that were significant in the univariate model were entered and adjusted for age. All statistical tests performed were 2 -sided, and a $p$ value $<0.05$ indicated significance. Statistical analyses were performed by using SPSS (https://www.ibm.com) and Stata version 15 (https://www.stata.com) software packages.

\section{Results}

Our study focused on oral HPV infections among the 331 infants born to the 329 mothers in the Finnish Family HPV Study cohort. The mean \pm SD age of the mothers at enrollment was $25.5 \pm 3.35$ years. Of the 
331 newborns, 5 did not participate in oral samplings at any visit, and 2 others had only 1 visit, resulting in a longitudinal cohort of 324 (171 girls and 153 boys) children (Figure). Participants had a follow-up mean \pm SD age of $51.9 \pm 28.9$ months (range 0.03-99.7 months). Of these children, $77.6 \%$ were born by vaginal delivery and $22.4 \%$ by cesarean section.

We collected general background information for the general health of the children recorded at the 36 -month visit as given by their parents (Table 1). Hand warts were reported only for 3 children, and a common childhood viral disease (molluscum contagiosum) was reported in for 18 children $(n=203)$. Allergy/atopic symptoms were identified in $26.6 \%$ of the children at the 36 -month visit. For $54 \%$ of the index families, the child was a firstborn. At the 6-year follow-up visit, $11 \%(20 / 180)$ of the children had clinical lesions on their oral mucosa. The most common lesions were small hyperplastic lesions (3.9\%), aphtous ulcers $(2.8 \%)$, and red lesions (2.2\%). Only 1 child had a papillary lesion; this child was positive for HPV16 at day 3 , month 1 , and month 24 and subsequently HPV negative at other visits. Hand warts at the time of examination were detected in 3\% of the children. There was no correlation recorded between the presence of hand warts and oral HPV at the 6-year visit.

We also provide an overview of oral HPV infections in children who had HPV genotypes and their point prevalence during the follow-up period (Table 1). The prevalence of oral HPV varied from $8.7 \%$ to $22.8 \%$ over time, and was lowest at the 3-year visit and highest at birth. Altogether, 18 different HPV genotypes were identified in the oral mucosa. HPV16 was the most prevalent genotype, followed by HPV18, 6, 33, and 31. The prevalence of multiple-type infections varied from $0.3 \%$ to $3.7 \%$. Overall, $22.9 \%$ of the oral samples collected immediately after birth were positive for HPV DNA. At that time point, the genotype distribution was also the widest (15 different HPV types), and the frequency of multiple-type infections was the highest (3.7\%). At the 36-month visit, only $8.7 \%$ of the oral samples were positive for HPV, and only 4 genotypes (HPV6, 11, 16, and 18) were identified. At the 6-year visit, HPV prevalence increased again to $20.4 \%$, and 8 different HPV genotypes were identified. A total of 25 different combinations of HPV co-infections (with $\geq 2$ genotypes) were recorded, HPV16 was present in $56 \%(14 / 25)$ of these samples. When analyzed by sex, we found differences in HPV prevalence at 1-, $2-, 12-$, and 36-month visits, but none at the 6-year visit. HPV positivity at birth or later was unrelated to the mode of delivery. Overall, $41.4 \%$ (135/329) of

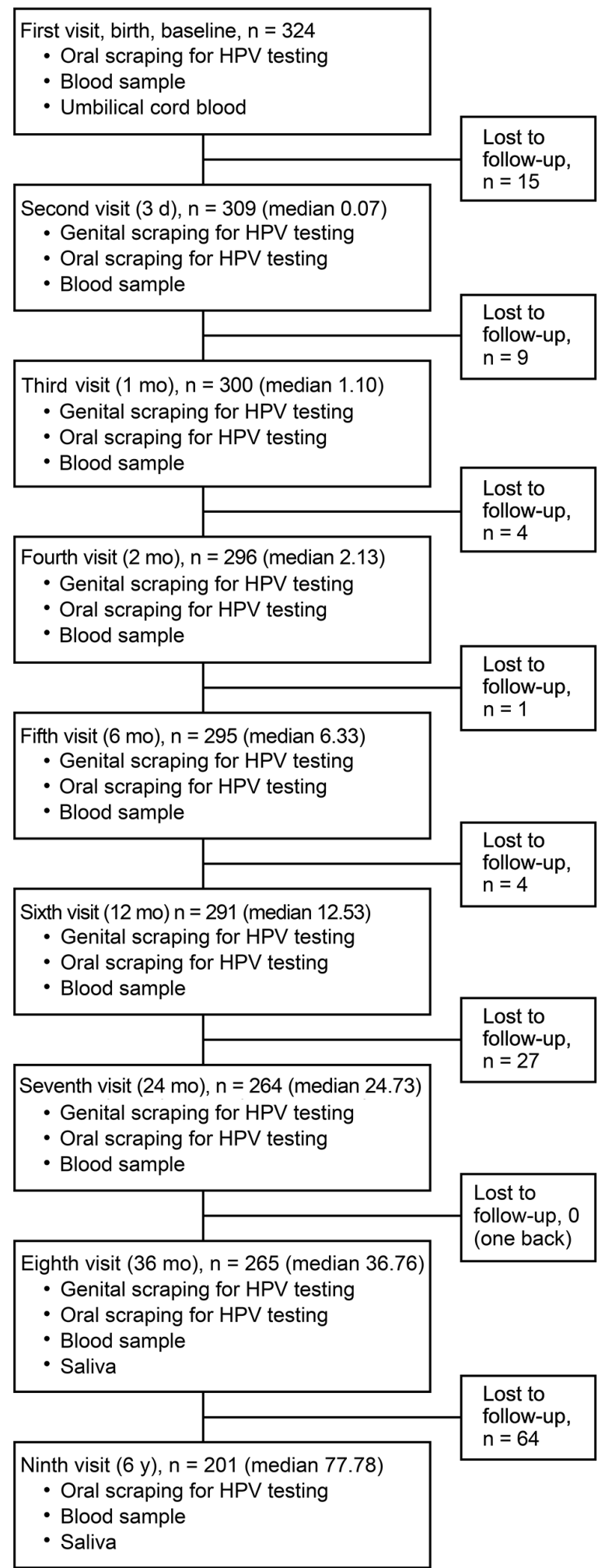

Figure. Oral HPV infection in 324 children in the Finnish Family HPV Study during the first 6 years of life. Each visit shows the number of children who participated in the specific follow-up, timeline of the visit, and samples obtained at each visit. HPV, human papillomavirus. 
Table 1. Prevalence and genotype variation of oral HPV infections among 324 children from birth to 6 y of age*

\begin{tabular}{|c|c|c|c|c|c|c|c|c|c|}
\hline Variable & At birth & $3 d$ & $1 \mathrm{mo}$ & $2 \mathrm{mo}$ & $6 \mathrm{mo}$ & $12 \mathrm{mo}$ & $24 \mathrm{mo}$ & $36 \mathrm{mo}$ & $6 y$ \\
\hline Oral sample & 324 & 309 & 300 & 296 & 295 & 291 & 264 & 265 & 201 \\
\hline Mean \pm SD age, mo & 0 & $\begin{array}{c}0.08 \pm \\
0.37\end{array}$ & $\begin{array}{c}1.14 \pm \\
0.18\end{array}$ & $\begin{array}{c}2.23 \pm \\
0.33\end{array}$ & $\begin{array}{c}6.41 \pm \\
0.45\end{array}$ & $\begin{array}{c}12.61 \pm \\
0.67\end{array}$ & $\begin{array}{c}24.90 \pm \\
1.01\end{array}$ & $\begin{array}{c}36.98 \pm \\
1.31\end{array}$ & $\begin{array}{c}77.47 \pm \\
11.01\end{array}$ \\
\hline Any HPV+ & $74(22.8)$ & $41(13.3)$ & $57(19)$ & $48(16.2)$ & $44(14.9)$ & $34(11.7)$ & $25(9.5)$ & $23(8.7)$ & $41(20.4)$ \\
\hline Girls, any HPV+ & 35 (47.9) & $19(46.3)$ & $25(43.9)$ & $29(60.4)$ & $20(46.5)$ & $20(58.5)$ & $14(56.0)$ & $10(43.5)$ & 20 (49.9) \\
\hline Boys, any HPV+ & $39(52.1)$ & $22(53.7)$ & $32(56.1)$ & 19 (39.6) & $24(53.5)$ & $14(51.5)$ & $9(44.0)$ & $13(56.5)$ & $21(51.2)$ \\
\hline Single HPV+ & 62 (19.1) & 36 (11.7) & 55 (18.3) & $44(14.8)$ & $36(12.2)$ & $33(11.0)$ & $20(7.6)$ & $21(7.9)$ & $35(17.4)$ \\
\hline Multiple HPV (>2) & $12(3.7)^{\prime}$ & $5(1.6)$ & $2(0.7)$ & $4(1.4)$ & $8(2.7)$ & $1(0.3)$ & $4(1.5)$ & $2(0.8)$ & $6(3.0)$ \\
\hline No. HPV genotypes & 15 & 5 & 8 & 7 & 9 & 10 & 7 & 4 & 8 \\
\hline \multicolumn{10}{|l|}{ Low-risk HPV } \\
\hline HPV6 & $11(3.4)$ & $5(1.6)$ & $3(1.0)$ & $7(2.4)$ & $4(1.4)$ & $3(1.0)$ & $1(0.4)$ & $2(0.8)$ & $4(2.0)$ \\
\hline HPV11 & $1(0.3)$ & - & $1(1.0)$ & $5(1.7)$ & $4(1.4)$ & $1(0.3)$ & - & $1(0.4)$ & - \\
\hline HPV70 & $2(0.6)$ & - & - & $1(0.3)$ & $1(0.3)$ & $1(0.3)$ & $2(0.8)$ & - & - \\
\hline \multicolumn{10}{|l|}{ High-risk HPV } \\
\hline HPV16 & $25(7.7)$ & $28(9.1)$ & $30(10)$ & $16(5.4)$ & $14(4.7)$ & $14(4.8)$ & $9(3.4)$ & $17(6.4)$ & $20(10)$ \\
\hline HPV18 & $4(1.2)$ & $1(0.3)$ & $9(3.0)$ & $11(3.7)$ & $7(2.4)$ & $8(2.7)$ & $4(1.5)$ & $1(0.4)$ & $2(1.0)$ \\
\hline HPV31 & $31(0.9)$ & - & - & $2(0.7)$ & - & $1(0.3)$ & - & - & $3(1.5)$ \\
\hline HPV33 & $2(0.6)$ & - & $8(2.7)$ & $2(0.7)$ & $1(0.3)$ & - & $2(0.8)$ & - & $2(1.0)$ \\
\hline HPV39 & $1(0.3)$ & - & $1(0.3)$ & - & - & - & - & - & $2(1.0)$ \\
\hline HPV45 & $1(0.3)$ & - & $1(0.3)$ & - & $1(0.3)$ & - & - & - & $1(0.5)$ \\
\hline HPV51 & - & - & - & - & $1(0.3)$ & $1(0.3)$ & - & - & - \\
\hline HPV52 & - & - & - & - & - & $1(0.3)$ & - & - & - \\
\hline HPV53 & $1(0.3)$ & $1(0.3)$ & - & & - & & - & - & - \\
\hline HPV56 & $3(0.9)$ & - & - & - & - & $1(0.3)$ & $1(0.4)$ & - & - \\
\hline HPV58 & $2(0.6)$ & - & - & - & - & $1(0.3)$ & - & - & $1(0.5)$ \\
\hline HPV66 & $4(1.2)$ & $1(0.3)$ & $2(0.7)$ & - & $1(0.3)$ & - & $1(0.4)$ & - & - \\
\hline HPV68 & $1(0.3)$ & - & - & - & - & - & - & - & - \\
\hline HPV82 & $1(0.3)$ & - & - & - & - & - & - & - & - \\
\hline
\end{tabular}

the children remained negative for all oral samples collected during the follow-up.

Incident HPV infection (baseline negative) was determined for 107 (32.8\%) of 326 children: 107 cases $/ 5,754$ person-months at risk (PMR), which resulted in an incidence rate of 18.6 cases $/ 1,000$ PMR. Ten children $(9.4 \%)$ had multiple-type infections among this group of incident infections. Kaplan-Meier analysis showed that there were no significant differences in the acquisition of oral HPV between the different species (Appendix Figure). The incidence of new HPV genotypes during the follow-up were also investigated by HPV clades. The results indicated that none of the HPV genotypes present at birth would promote acquisition of another specific HPV genotype, not even an HPV from the same clade. However, newborns with oral HPV6 or HPV11 ( $\mathrm{n}=4)$ acquired only HPV16 or HPV18 genotypes. We provide the type distribution of children who were positive at 6-year visit across different time points (Appendix Table 2). The results showed that $63 \%(26 / 41)$ had the same genotype detectable already at birth, and $14.6 \%$ (6/41) of the children had the same genotype at some visit during the follow-up, but not at birth. Of the children, $22 \%(9 / 41)$ had the genotype present only at most recent (6-year) follow-up visit. Four of the children positive for HPV6 at birth still had this genotype at their 6-year follow-up visit.

A total of 99 children cleared their oral HPV infection during the follow-up, resulting in a clearance rate of 19.1 cases/1,000 PMR (99/5,183). The mean clearance times for clades 10 (HPV6/11 and their closest relative), 9 (HPV16 group), and 7 (HPV18) genotypes were not significantly different: 28.6, 34.2, and 30 months, respectively (Appendix Figure).

A total of $14.9 \%(48 / 323)$ of the children had persistent oral HPV infection. The mean time of persistence was 20.6 months (range 0.1-92.2 months). We provide type-specific HPV persistence times (Table 2). The most prevalent type to persist was HPV16, which had a persistence time of 19.8 months, followed by multiple-type infections (persistence time 14.2 months), HPV18 (persistence time 11.8 months), HPV33 (persistence time 14.2 months), and HPV6 (persistence time 19.7 months). The 6 children who had multiple-type HPV infections at birth still had them at the most recent visit. Our results show that clade a9 resulted most frequently in the full-time persistence of oral HPV infection in early childhood, followed by clade a7.

We summarized the predictors of incident, cleared, and persistent oral high-risk HPV infections in these children (Table 3). All established or implicated 
Table 2. Duration of genotype and species-specific persistence of oral HPV infection in children*

\begin{tabular}{lcc}
\hline HPV genotypes/clades & No. & Mean persistence, mo (range) \\
\hline HPV6 & 2 & $19.7(1.8-37.5)$ \\
HPV16 & 36 & $19.8(0.1-82)$ \\
HPV18 & 3 & $11.8(5.0-18.6)$ \\
HPV31 & 1 & $92.2(92.2)$ \\
HPV33 & 3 & $14.2(1.0-40.6)$ \\
HPV39 & 1 & $89.0(89.0)$ \\
HPV58 & 1 & $88.7(88.7)$ \\
Multiple-type infections $(\geq 2)$ & 20 & $14.2(1.0-91.0)$ \\
Clade A7: HPV18, 39, 45, 59, 68, 70, 85 & 4 & $31.2(5.0-89.0)$ \\
Clade A9: HPV16, 31, 33, 35, 52, 58 & 41 & $22.8(0.1-92.2)$ \\
Clade A10: HPV6, 11, 13, 44, 55, 74 & 2 & $19.7(1.8-37.7)$ \\
\hline${ }^{*}$ Persistence is defined as having $\geq 2$ consecutive visit HPV-positive results for the same HPV genotype or clade. HPV, human papillomavirus.
\end{tabular}

risk factors in our previous studies were tested as covariates, but we report only those that showed any significant predictive value (Table 3). Demographic data obtained at the 36-month visit for children did not show any association for oral HPV (Appendix Table 1). High-risk HPV seropositivity was associated with oral high-risk HPV incidence for fathers and clearance for children. Incidence rates were 3.32 (95\% CI 1.24-8.91) for fathers and 5.84 (95\% CI 2.09-16.32) for children. Conversely, baseline oral carriage for mothers, as well as high-risk HPV seroconversion, were associated with persistent oral high-risk HPV infection for children. Odds ratios were 1.92 (95\% CI 1.35-2.74) for baseline oral carriage and 1.60 (95\% CI 1.02-2.50) for high-risk HPV seroconversion.

\section{Discussion}

HPV infections in the oral cavity have been detected in young children, but the outcome of these infections has remained unknown. We found that the prevalence of HPV and multiple-type infections was highest and the spectrum of HPV genotypes was widest at birth. The mode of delivery had no association with oral HPV carriage, and some sex differences were found in oral HPV prevalence during the early months, but not at the end of the follow-up period. Results indicate that none of the HPV genotypes present at birth would promote acquiring another specific HPV genotype, not even an HPV from the same clade. Although most of the oral HPV infections were cleared during the 6-year follow-up period, persistent oral HPV infection was found in $14.9 \%$ of these children. The 6 children who had multiple-type HPV infections at birth still harbored those infections at the most recent visit. Thus, clade a9 resulted most frequently in the full-time persistence of oral HPV infection during the early childhood, followed by a7 as the second most frequent clade.

HPV acquisition at birth has been regarded to be caused by vertical transmission, although controversial opinions have been reported $(3,5,6)$. The debate is ongoing, particularly regarding the magnitude of risk, as well as route and timing, and whether mother-to-child transmission of HPV is a major infection route. Neonatal HPV infection through vertical transmission is believed to be transient, although there have been only a few follow-up studies $(5,11,12,22)$. One of those studies showed that 37\% (39/106) of

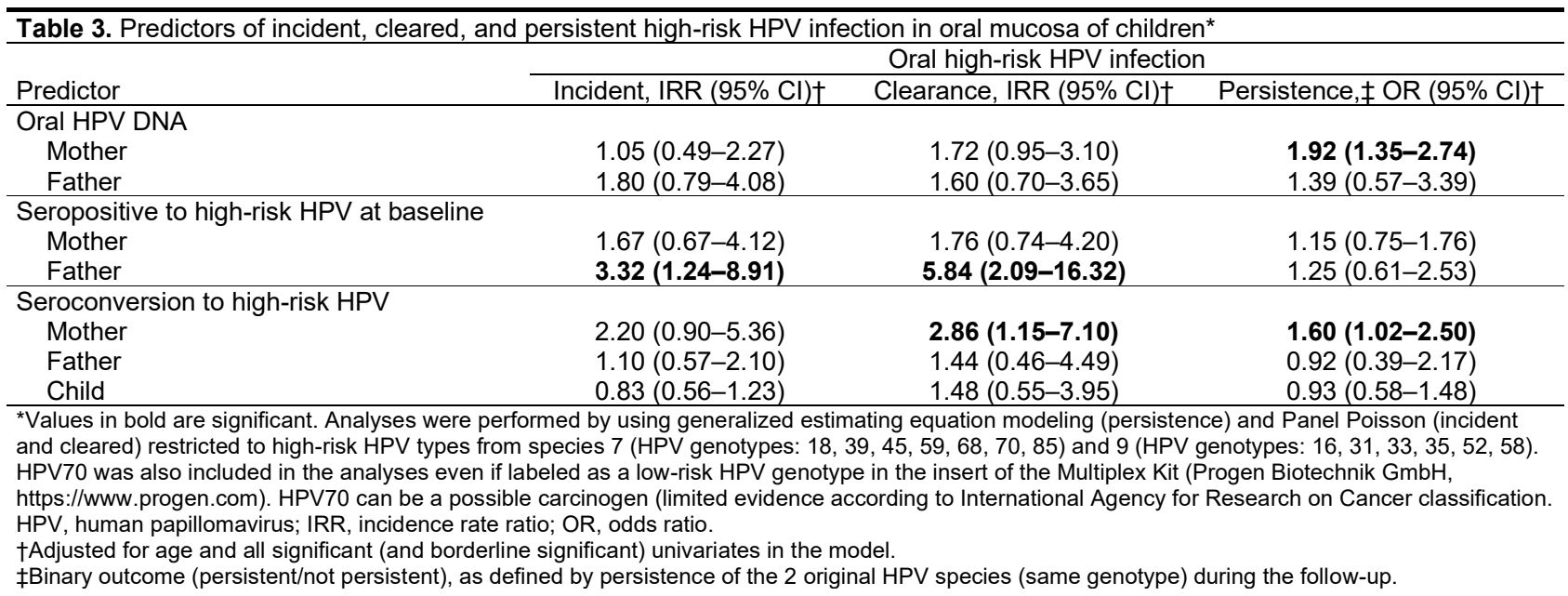


nasopharyngeal aspirates of newborns were HPV positive, and concordance between HPV types in the mother (genital tract) and newborn was 69\% (12). In a few days, HPV positivity disappeared in $38 \%$ of these newborns. However, $10.4 \%$ of the infants had the same HPV type detectable at birth and after 3 months to 3 years (12). Another study reported that 32\% (31/98) of the children (age range 3.6 months-11.6 years) born to mothers who had cervical HPV infections at the time of delivery had HPV detectable in their oral mucosa (11). A total of $52 \%$ of these children had an HPV type identical with that of their mothers; HPV16/18 was most prevalent (81\%).

Our results are consistent with previous results because they show that oral HPV is detectable in $22.8 \%$ of newborns. This HPV detection rate is almost identical to what Castellsague et al. reported in 2009, showing that the overall oral HPV prevalence at any visit was $18.2 \%$ during the mean follow-up of 14 months, by using PCR (MY09/MY11 primers), followed by subsequent hybridization with specific probes for HPV6, 11, 16, 18, 31, 33, and 39 (22). That study reported HPV positivity of $12.7 \%(14 / 110)$ at 6 weeks of age; in our study, HPV positivity at month 2 was $16.2 \%(48 / 296)$. Similar to our present results, HPV16 was the most frequent genotype, followed by HPV6/11, HPV18, and HPV31 (22) and in this study by HPV18, 6, 31, and 33 .

Our results are derived from a longitudinal study rather than a cross-sectional study, in which autocorrelation (intrasubject variability) has been controlled by models for panel data (GEE and panel Poisson). Trottier et al. published their first results from the HERITAGE study on perinatal transmission and risk for HPV persistence in children (23). The design of their study is nearly identical with that of our study, but they extended sampling to conjunctival, pharyngeal, and genital sites. Their preliminary results on $75 \mathrm{HPV}$-positive participating mothers and their 67 infants sampled at birth and at 3-month visits showed that overall HPV positivity in children was $11 \%$ (range 5\%-22\%). Site-specific HPV positivity for conjunctival and genital areas were $4.8 \%$ and $4.8 \%$, respectively (23). However, the HPV detection rate was only $8.1 \%$ for oral sites and $1.6 \%$ for oropharyngeal sites, which was lower than that reported in our study. Oral sampling (brushing of the entire oral mucosa vs. Dacron swab of buccal mucosa) and HPV amplification (nested vs. single PCR) might explain the differences in oral HPV detection rates between these 2 studies.

HPV data for mothers were not included in our report because these data have been reported in other studies $(14,20,24,25)$. In brief, HPV DNA was detected in $17.9 \%$ of baseline oral samples from newborns and in $16.4 \%$ of maternal cervical samples (14). The HPV genotype-specific concordance between the newborns at delivery and the mothers was almost perfect (weighted $\kappa=0.988 ; 95 \%$ CI $0.951-0.997$ ), but this concordance disappeared in 2 months (14). We have also shown that oral HPV carriage in newborns was most significantly associated with HPV presence in the placenta or cord blood $(9,14)$. Together with these previous baseline data, our study strongly supports the hypothesis that HPV can be transmitted vertically and cause a true infection of oral mucosa of the newborn. Some of these oral HPV infections acquired at birth can also persist for years without any major clinical lesions. We reported the detection of HPV16specific cell-mediated immunity in a small number of sexually inexperienced children from this cohort $(25,26)$. However, we cannot determine by detection of HPV DNA the time when HPV-evoked immune recognition occurred. It has also been shown that half of healthy adults demonstrate HPV-specific cell-mediated immunity, irrespective of their partner/sexual status $(27,28)$.

Oral HPV persistence during the 6-year followup period was predicted by oral HPV infection and seroconversion to high-risk HPV of the mother during the follow-up. We have recently shown that human leukocyte antigen $G$ has a role in predicting the likelihood of the newborn for oral HPV infection at birth (29). However, human leukocyte antigen G had no association with HPV genotype-specific concordance between the mother and her child at birth or influence on perinatal HPV status of the child. This finding suggests that some persistent oral HPV infections after birth are not caused by vertical transmission but are acquired horizontally from the mother. This finding also indicates that transmission from a mother to her child continues during early childhood. In addition, some of the so-called persistent HPV infections could be reinfections among the family, and the incident HPV infections for a child were predicted by HPV seropositivity of the father. However, reinfection of the child needs to be further studied.

In our study, $41 \%$ of children remained constantly HPV negative during the follow-up period ( $\leq 9$ consequent oral samples). We do not know yet whether these children will continue to remain HPV negative later in life. We suspect that these children might be less prone to HPV infections in general, and would be interesting to evaluate again later in life.

In conclusion, our results indicate that HPV infection can be acquired nonsexually and is already 
common at an early age. The oral cavity is the common site of the first HPV exposure, and the mother is the most likely source of first HPV infection in her child. These results have several major implications in HPV vaccination programs. If a subgroup of children can acquire a persistent HPV infection, the timing of prophylactic HPV vaccination is imperative. Maternal HPV antibodies, irrespective of whether they are acquired by natural HPV infection or vaccination, might protect the fetus, newborn, and young child against early HPV infection. In addition, children who have persistent HPV infections (caused by immunologic tolerance) might also benefit from vaccination, as has been the case with hepatitis B virusinfected newborns or children (30).

\section{Acknowledgments}

We thank Elisa Hovimäki for participating in the enrollment of the women in the Finnish Family HPV study and their clinical follow-up and Tatjana Peskova, Mariia Valkama, and Ketlin Adel for providing skillful technical assistance.

This study was supported by the Academy of Finland (grants 116438/2006 and 0204/2008), the Finnish Cancer Foundation, and the Sohlberg Foundation.

\section{About the Author}

Dr. Stina Syrjänen is a professor and chairman emerita in the Department of Oral Pathology, Faculty of Medicine, University of Turku, Turku, Finland, and chief physician in the Department of Pathology, Turku University Hospital. Her primary research interests are HPV, its transmission mode, and its role in early carcinogenesis.

\section{References}

1. Weissenborn SJ, De Koning MN, Wieland U, Quint WG, Pfister HJ. Intrafamilial transmission and family-specific spectra of cutaneous betapapillomaviruses. J Virol. 2009;83:811-6. https://doi.org/10.1128/JVI.01338-08

2. Rombaldi RL, Serafini EP, Mandelli J, Zimmermann E, Losquiavo KP. Perinatal transmission of human papilomavirus DNA. Virol J. 2009;6:83. https:/ / doi.org/ 10.1186/1743-422X-6-83

3. Syrjänen S. Current concepts on human papillomavirus infections in children. APMIS. 2010;118:494-509. https://doi.org/10.1111/j.1600-0463.2010.02620.x

4. D'Souza G, Kluz N, Wentz A, Youngfellow RM, Griffioen A, Stammer E, et al. Oral human papillomavirus (HPV) infection among unvaccinated high-risk young adults. Cancers (Basel). 2014;6:1691-704. https:// doi.org/10.3390/ cancers6031691

5. Liu Z, Rashid T, Nyitray AG. Penises not required: a systematic review of the potential for human papillomavirus horizontal transmission that is non-sexual or does not include penile penetration. Sex Health. 2016;13:10-21. https://doi.org/10.1071/SH15089
6. Merckx M, Liesbeth WV, Arbyn M, Meys J, Weyers S, Temmerman M, et al. Transmission of carcinogenic human papillomavirus types from mother to child: a meta-analysis of published studies. Eur J Cancer Prev. 2013;22:277-85. https://doi.org/10.1097/ CEJ.0b013e3283592c46

7. de Martel C, Plummer M, Vignat J, Franceschi S. Worldwide burden of cancer attributable to HPV by site, country and HPV type. Int J Cancer. 2017;141:664-70. https://doi.org/10.1002/ijc.30716

8. Chatzistamatiou K, Sotiriadis A, Agorastos T. Effect of mode of delivery on vertical human papillomavirus transmission: a meta-analysis. J Obstet Gynaecol. 2016;36:104. https:// doi.org/10.3109/01443615.2015.1030606

9. Sarkola ME, Grénman SE, Rintala MA, Syrjänen KJ, Syrjänen SM. Human papillomavirus in the placenta and umbilical cord blood. Acta Obstet Gynecol Scand. 2008;87:1181-8. https:/ / doi.org/10.1080/00016340802468308

10. Smith EM, Parker MA, Rubenstein LM, Haugen TH, Hamsikova E, Turek LP. Evidence for vertical transmission of HPV from mothers to infants. Infect Dis Obstet Gynecol. 2010;2010:326369. https:// doi.org/10.1155/2010/326369

11. Puranen M, Yliskoski M, Saarikoski S, Syrjänen K, Syrjänen S. Vertical transmission of human papillomavirus from infected mothers to their newborn babies and persistence of the virus in childhood. Am J Obstet Gynecol. 1996;174:694-9. https://doi.org/10.1016/S0002-9378(96) 70452-0

12. Puranen MH, Yliskoski MH, Saarikoski SV, Syrjänen KJ, Syrjänen SM. Exposure of an infant to cervical human papillomavirus infection of the mother is common. Am J Obstet Gynecol. 1997;176:1039-45. https:/ / doi.org/10.1016/ S0002-9378(97)70399-5

13. Rintala MA, Grénman SE, Puranen MH, Isolauri E, Ekblad U, Kero PO, et al. Transmission of high-risk human papillomavirus (HPV) between parents and infant: a prospective study of HPV in families in Finland. J Clin Microbiol. 2005;43:376-81. https://doi.org/10.1128/ JCM.43.1.376-381.2005

14. Koskimaa HM, Waterboer T, Pawlita M, Grénman S, Syrjänen K, Syrjänen S. Human papillomavirus genotypes present in the oral mucosa of newborns and their concordance with maternal cervical human papillomavirus genotypes. J Pediatr. 2012;160:837-43. https://doi.org/ 10.1016/j.jpeds.2011.10.027

15. Laprise C, Trottier H, Monnier P, Coutlée F, Mayrand MH. Prevalence of human papillomaviruses in semen: a systematic review and meta-analysis. Hum Reprod. 2014;29:640-51. https://doi.org/10.1093/humrep/det453

16. Aagaard K, Ma J, Antony KM, Ganu R, Petrosino J, Versalovic J. The placenta harbors a unique microbiome. Sci Transl Med. 2014;6:237ra65. https:/ / doi.org/10.1126/ scitranslmed.3008599

17. Zouridis A, Kalampokas T, Panoulis K, Salakos N, Deligeoroglou E. Intrauterine HPV transmission: a systematic review of the literature. Arch Gynecol Obstet. 2018;298:35-44. https:/ / doi.org/10.1007/s00404-018-4787-4

18. Louvanto K, Rintala MA, Syrjänen KJ, Grénman SE, Syrjänen SM. Genotype-specific persistence of genital human papillomavirus (HPV) infections in women followed for 6 years in the Finnish Family HPV study. J Infect Dis. 2010;202:436-44. https:/ / doi.org/10.1086/653826

19. Schmitt M, Bravo IG, Snijders PJ, Gissmann L, Pawlita M, Waterboer T. Bead-based multiplex genotyping of human papillomaviruses. J Clin Microbiol. 2006;44:504-12. https://doi.org/10.1128/JCM.44.2.504-512.2006 
20. Syrjänen S, Waterboer T, Sarkola M, Michael K, Rintala M, Syrjänen $\mathrm{K}$, et al. Dynamics of human papillomavirus serology in women followed up for 36 months after pregnancy. J Gen Virol. 2009;90:1515-26. https:/ / doi.org/ 10.1099/vir.0.007823-0

21. Waterboer T, Sehr P, Michael KM, Franceschi S, Nieland JD, Joos TO, et al. Multiplex human papillomavirus serology based on in situ-purified glutathione s-transferase fusion proteins. Clin Chem. 2005;51:1845-53. https:/ / doi.org/ 10.1373/clinchem.2005.052381

22. Castellsagué X, Drudis T, Cañadas MP, Goncé A, Ros R, Pérez JM, et al. Human papillomavirus (HPV) infection in pregnant women and mother-to-child transmission of genital HPV genotypes: a prospective study in Spain. BMC Infect Dis. 2009;9:74. https:/ /doi.org/10.1186/1471-2334-9-74

23. Trottier $\mathrm{H}$, Mayrand MH, Coutlée F, Monnier $\mathrm{P}$, Laporte L, Niyibizi J, et al. Human papillomavirus (HPV) perinatal transmission and risk of HPV persistence among children: design, methods and preliminary results of the HERITAGE study. Papillomavirus Res. 2016;2:145-52. https://doi.org/10.1016/j.pvr.2016.07.001

24. Rintala MA, Grénman SE, Järvenkylä ME, Syrjänen KJ, Syrjänen SM. High-risk types of human papillomavirus (HPV) DNA in oral and genital mucosa of infants during their first 3 years of life: experience from the Finnish HPV Family study. Clin Infect Dis. 2005;41:1728-33. https:// doi.org/10.1086/498114

25. Koskimaa HM, Paaso AE, Welters MJ, Grénman SE, Syrjänen $\mathrm{KJ}$, van der Burg $\mathrm{SH}$, et al. Human papillomavirus 16 E2-, E6- and E7-specific T-cell responses in children and their mothers who developed incident cervical intraepithelial neoplasia during a 14-year follow-up of the Finnish Family HPV cohort. J Transl Med. 2014;12:44. https:/ / doi.org/ 10.1186/1479-5876-12-44

26. de Jong A, van der Burg SH, Kwappenberg KM, van der Hulst JM, Franken KL, Geluk A, et al. Frequent detection of human papillomavirus $16 \mathrm{E} 2$-specific T-helper immunity in healthy subjects. Cancer Res. 2002;62:472-9.

27. Koskimaa HM, Paaso A, Welters MJ, Grénman S, Syrjänen K, van der Burg SH, et al. Human papillomavirus 16-specific cell-mediated immunity in children born to mothers with incident cervical intraepithelial neoplasia (CIN) and to those constantly HPV negative. J Transl Med. 2015;13:370. https:/ /doi.org/10.1186/s12967-015-0733-4

28. Welters MJ, de Jong A, van den Eeden SJ, van der Hulst JM, Kwappenberg KM, Hassane S, et al. Frequent display of human papillomavirus type 16 E6-specific memory t-Helper cells in the healthy population as witness of previous viral encounter. Cancer Res. 2003;63:636-41.

29. Louvanto K, Roger M, Faucher MC, Syrjänen K, Grenman S, Syrjänen S. HLA-G and vertical mother-to-child transmission of human papillomavirus infection. Hum Immunol. 2018; 79:471-6. https://doi.org/10.1016/ j.humimm.2018.03.002

30. Qu C, Chen T, Fan C, Zhan Q, Wang Y, Lu J, et al. Efficacy of neonatal HBV vaccination on liver cancer and other liver diseases over 30-year follow-up of the Qidong hepatitis B intervention study: a cluster randomized controlled trial. PLoS Med. 2014;11:e1001774. https:/ / doi. org/10.1371/journal.pmed.1001774

Address for correspondence: Stina Syrjänen, Department of Oral Pathology and Oral Radiology, Institute of Dentistry, Faculty of Medicine, University of Turku, Lemminkäisenkatu 2, 20520

Turku, Finland; e-mail: stisyr@utu.fi

\section{EID Podcast} Enterovirus D68 and Acute Flaccid Myelitis, 2020

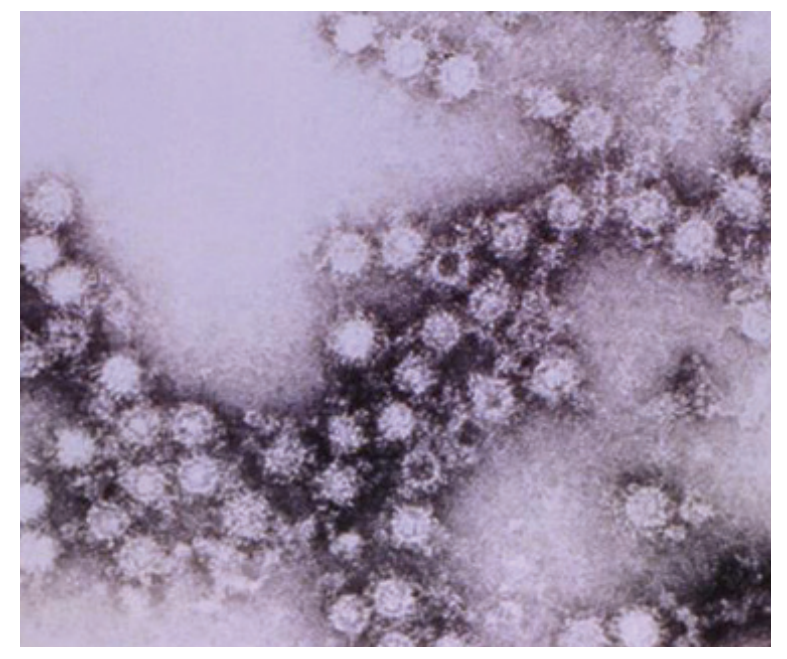

Around 2014, a mysterious, polio-like illness emerged in California and Colorado. Acute flaccid myelitis (AFM) primarily infects children, and if untreated, can lead to paralysis and respiratory failure. Despite extensive surveillance and research campaigns, the true cause of this debilitating disease remains unknown.

New research has shed light on a possible connection between AFM and a pathogen called enterovirus D68.

In this EID podcast, Dr. Sarah Kidd, a medical epidemiologist at CDC, and Sarah Gregory discuss what is known-and unknownabout AFM.

\section{Visit our website to listen:} https: / / go.usa.gov/x7CkY EMERGING INFECTIOUS DISEASES ${ }^{\circ}$ 